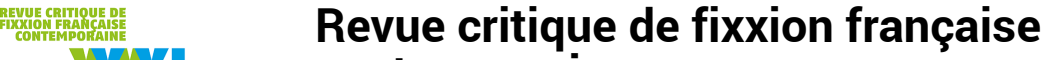 \\ XXI contemporaine

22 | 2021

Figures du mensonge et de la mauvaise foi dans le roman contemporain

\section{Vie de Cléobule, hérésiarque}

\section{Pierre Senges}

\section{(2) OpenEdition}

\section{Journals}

Édition électronique

URL : https://journals.openedition.org/fixxion/381

DOI : 10.4000/fixxion.381

ISSN : 2295-9106

Éditeur

Ghent University

\section{Référence électronique}

Pierre Senges, «Vie de Cléobule, hérésiarque », Revue critique de fixxion française contemporaine [En ligne], 22 | 2021, mis en ligne le 15 juin 2021, consulté le 17 février 2022. URL : http://

journals.openedition.org/fixxion/381; DOI : https://doi.org/10.4000/fixxion.381

Ce document a été généré automatiquement le 17 février 2022.

\section{(c) () $\odot$}

Les contenus de la Revue critique de fixxion française contemporaine sont mis à disposition selon les termes de la licence Creative Commons Attribution - Pas d'Utilisation Commerciale - Pas de Modification 4.0 International 


\title{
Vie de Cléobule, hérésiarque
}

\author{
Pierre Senges
}

1 En Asie Mineure ou à Alexandrie, au second siècle de notre foi, quand Basilide faisait de l'univers une improvisation téméraire et mal intentionnée d'anges déficients, Cléobule ambitionnait, avec une singulière détermination, de figurer au catalogue des hérésiarques mineurs - ou même majeurs, après tout. Il y pensait la nuit, au cours de rêves envoyés par un dieu exagérément flatteur, déjà absconditus et trompeur à sa manière (chacun son style); il en rêvait le jour et son rêve prenait alors la forme de démonstrations solides et sans relâche faites en présence d'un seul auditeur vite épuisé (selon une expression empruntée à un imitateur d'Horace, il avait tôt fait de vider son sac d'écoute) ; il en rêvait au cours de périodes intermédiaires, androgynes, ni jour ni nuit, de grasses matinées passant pour méditatives, pour le comble d'une retraite studieuse.

2 On ignore la date exacte de sa naissance : la connaître serait suspect si l'on songe à toutes ces approximations d'état civil au second siècle, les bibliothèques parties en fumée, les papyrus reconvertis en bourre de selle et les agents de l'administration essentiellement consacrés au service de l'annone, à la distribution des soldes pour des mercenaires perdus au fin fond d'un désert où Rome a réussi à planter son enseigne. On admet en général, par courtoisie, qu'il voit le jour "quelque part" vers Pergame et "quelque part" vers 100 après Jésus-Christ, mais l'une et l'autre de ces précisions imprécises semblent être le résultat d'un pari fait un soir de Côtes de Gascogne entre un spécialiste et un autre spécialiste, à l'aide d'une carte Michelin et d'une paire de dés l'élément le plus authentiquement romain. Peu importe, finalement, Pergame ou Sinope ou l'une ou l'autre des Troies éparpillées partout à l'est d'Istanbul, là où il y a des restes de murailles : on voit apparaître Cléobule pour la première fois sur un âne, comme Jésus à son époque, jeune encore, plus ou moins beau selon des critères désormais révolus, imberbe, le teint clair, presque blafard par contraste avec le gris fusain uni de son âne, et maigre avec de gros genoux comme ceux d'un adolescent, les os des omoplates si fins, paraît-il, “on voyait le jour à travers" (Clément d'Alexandrie, Stromateis). Plus tard, à Rome, en route vers Rome puis hors de Rome en la fuyant à bride abattue, sur le dos d'un âne, à la fois le même et un autre, revoilà cléobule ; les témoins croient le reconnaître, au teint mat presque noir comparé à la blancheur de 
son âne, aux cuisses fortes comme s'il avait gravi pour le plaisir cent fois de suite le Golgotha, et une barbe d'un jour (l'impossibilité de se raser comme l'impossibilité de faire lever le pain le jour de la pâque juive).

Marcion, Carpocrate, Valentin, Arius, Mani et Pélage ont connu, connaissent ou connaîtront d'ici peu une gloire enviable, de traînée de poudre, de comète, de prodige, de razzia et d'échauffourée, d'engouement soudain, universel à l'échelle d'une moitié de continent ou d'une province, peut-être retombée dix ans plus tard pour devenir une légende plus terne, archivée dans quelque ouvrage et lue par des clercs, avec méfiance, mais toujours séduisante. Cléobule aurait pu secréter dans sa chambre d'adolescent des rêves de grand capitaine, pourquoi pas Alexandre, le soleil et l'ombre, les langues étrangères, les yeux de Peter O'Toole : mais pour être Alexandre, encore faut-il être le fils de Philippe de Macédoine, pas celui de Glaucon, meunier tombé un jour dans sa farine et pleuré par Xanthia - alors, au lieu d'un destin façon grand Alexandre jusqu'à l'Indus, et après l'Indus une forme de supplément de vie dans une portion de ciel entre le Cygne, l'Aigle et la Couronne Boréale, le petit Cléobule avec ses omoplates translucides, ses genoux comme deux courgettes de Nice, envisage une gloire d'hérésiarque.

4 L'hérésie, voilà l'or et l'argent d'un destin de blanc-bec de province, héritier d'un pétrin vide : l'unique façon de s'arracher à une routine établie d'avance, à une prédestination mesquine, sans fantaisie, au chômage, à des déterminismes supportés comme des sortilèges. Au lieu d'une petite vie de meunier menée à petits pas en portant des sacs lourds suivis par des charançons, mieux vaut en effet l'existence flamboyante de l'hérésiarque : solitaire, mais d'une solitude solaire, pas médiocre ni fortuite, en chemin vers un héroïsme singulier, bientôt même une sainteté de mauvais garçon tout aussi lumineuse, crapahuteuse et batailleuse, ornée de combats gagnés et perdus (les combats perdus plus bénéfiques encore que les combats gagnés, s'ils ajoutent la gloire du génie incompris de son vivant à la beauté des passes d'armes). Il aura pour lui la gloire de l'étude, la gloire des harangues, de la conspuation, c'est-à-dire de luttes à mains nues, sans cesse reportées au lendemain, contre toutes les figures du pouvoir en place, apôtres ou apôtres d'apôtres, grands fauves de l'Église entourés de disciples à la fois mâles et femelles à qui ils récitent sans se lasser leur rencontre avec l'homme qui avait connu le Christ en personne. Gloire de l'élucubration, de la prophétie, de la réfutation des erreurs du passé, du millénarisme reconduit tous les premiers de l'an, splendeur de l'hérésiarque au discours décousu, d'autant plus admirable, à charge pour les disciples de tisser quand leur tour viendra des liens entre le coq et l'âne, et gloire radieuse de l'hérésiarque découvreur d'un secret nouveau, assez bon pour le partager avec le reste de l'humanité, pourvu qu'elle le mérite.

5 Ah, il n'y a rien à dire : une vie exaltante, ce qu'on pourrait définir depuis notre $\mathrm{XXI}^{\mathrm{e}}$ siècle comme la synthèse d'une vie de star du punk, de vedette de cinéma, de philosophe médiatique un peu voyou mais tenté par l'Académie, à la fois penseur reclus (le charme de l'ermitage, le silence d'Ambroise espionné par Augustin) et meneur de foule (les acclamations, les évadés de la mer Rouge). Sans compter, en plus de tout ça, les honneurs de l'indiscipline : tout ce qui condamnait un homme jusque-là se renverse sous l'effet de l'hérésie (l'hérésie comme sacerdoce et comme genre littéraire): l'ignorance devient table rase et candeur de l'enfance, le désordre devient profusion, le confus devient richesse délivrée en vrac, l'incohérence devient préface au corpus hermétique, l'inconstance devient preuve d'une liberté sans égale, la paresse devient 
économie de moyen, les poncifs deviennent les sept piliers de la sagesse, la trahison est un geste de rupture et la rupture l'emblème du courage ("le jaune d'œuf du courage dans le blanc de l'univers poltron"), l'élucubration est la pensée en train de se faire et les prophéties ratées exigent des disciples une forme plus raffinée de foi.

6 Une certitude, pour Cléobule, vivace dès l'aube comme une envie soudaine, encore valable le soir, preuve sans doute de sa solidité (l'acharnement à défaut de la pertinence) : le devoir de tout homme sur Terre est de fonder une religion à son image puis d'entraîner sur cet élan la moitié du genre humain, toute la beauté de l'exercice consistant à faire de son idiosyncrasie une ferveur universelle - chacun des mortels de ce monde pourra se subsumer ensuite sous la catégorie d'un unique Moi. Cette certitude a longtemps été chez lui un bon prétexte pour se lever le matin : qui veut quitter son matelas doit rendre le monde extérieur plus enviable que le lit, faute de quoi le lit l'emportera toujours sur le monde : ça passe alors (du moins, Cléobule passe) par des raisonnements, des plaidoiries en faveur du monde, par l'énumération de ses vertus, ses maigres délices comparées au moelleux du lit, un moelleux protecteur, pacifique, érotique et méditatif. On y arrive bon an mal an, chacun trahit son désir de grasse matinée en se fouettant comme il peut la raison, en se donnant, comme la veille et comme le lendemain, l'illusion d'embrasser bientôt un monde enviable, fait d'intelligentes saveurs.

7 Il faut savoir courir sans doute, quand on est hérésiarque (ou, pour emprunter l'expression de Juvénal, qui l'avait lui-même empruntée à Empédocle : savoir prendre ses cliques et ses claques) : déménager à tout bout de champ, convertir l'Europe, des Colonnes d'Hercule à la Corne d'Or, en un vaste territoire de l'impermanence où les hérétiques dans son genre, téméraires un jour, timorés le jour suivant, se croisent sur les routes en faisant mine de ne pas se reconnaître. (La prudence sans doute, une stratégie d'espions dans le hall de l'hôtel Crillon à Paris, mais il faut aussi ajouter : ils se détestent, leur renommée est un combat carillonnant contre le renom des autres, leur foi absolue tournée vers l'Un universel est aussi affaire de concurrence. Faire défection, quitter l'Église de Rome encore à ses débuts, rien de plus simple, provoquer un schisme, la belle affaire, il suffit d'un peu d'endurance et le sens de l'opportunité, un talent de gratte-cul ou du génie précoce, Mozart au tabouret, tout aussi agaçant, souvent mal employé - mais affronter d'autres hérésiarques sur un même terrain, voilà une tâche abominable.)

8 C'est un beau rêve, le parcours de l'hérésiarque à vingt ans depuis un désert, où il recrute deux, trois disciples cueilleurs de figues, jusqu'aux portes de Rome, où il pourra narguer quelques figures d'autorité plus ou moins bien établies, sans les dorures du Vatican mais déjà l'aplomb ecclésiastique - entre temps, il aura provoqué un concile ou deux sur les sujets de son choix, il aura vu des docteurs s'arracher les cheveux et d'autres sans cheveux tenter de trouver chez les petits prophètes la réponse à ses questions oiseuses. Mais ça ne marche pas comme prévu, Cléobule arpente son désert sans trouver de cueilleurs de figues ni de rempailleur de chaises, et quand il en trouve, il ne leur fait pas découvrir la lumière, on dirait même qu'il les assomme, le rempailleur retourne à sa chaise, il paraît puiser davantage de bienfaits dans sa paille que dans ces discours où il est question d'hypostases. Quand cléobule finit par recruter de force, "en grattant le fond d'une marmite", un chevrier, un porcher, une veuve sept fois veuve et un orphelin sourd, il a du mal à les mettre en marche, du mal aussi à les nourrir tous les 
jours, et son enseignement au sujet de l'inconnaissance de Dieu selon Job leur passe, comme disait Démétrios de Phalère, largement au-dessus de la tête.

De sa doctrine, d'ailleurs, il faut bien le reconnaître, on ne sait pas grand-chose : on rassemble des morceaux épars sauvés du naufrage, de tous les déluges et du pilon des éditeurs - de la même façon, certains téméraires s'acharnent à agiter les trois fragments de Pericon l'Abscons ("infini", "ongle" et "j'ai parfois l'impression que") pour en déduire le fond de sa philosophie (il y a aussi les trois fragments authentifiés d'Anaximène : "bonnet", "clou" et "être suspendu"). L'essentiel nous est connu par ses pires ennemis : qui ont pris soin de préserver ses fragments les plus notables, les plus frappants et donc les plus faibles, sous forme de citations dans leurs propres ouvrages, pas très nombreux à vrai dire, recopiés de siècle en siècle par des clercs prudents c'était pour en rire à tête reposée, entre amis, ça n'a pas empêché cléobule de leur être reconnaissant, tout content de voir survivre dans les pages des autres les meilleures bribes de ses Vérités nouvelles et finales (vite disparues dans l'incendie d'une épicerie de quartier, à Syracuse). Pour l'essentiel, ce qu'on en retient : une interprétation elle aussi téméraire et mal intentionnée du Cantique des Cantiques, une surévaluation du rôle de Putiphar, l'ajout d'une cinquième couche d'interprétation des textes, en plus des interprétations littérale, allégorique, tropologie et anagogique, tenant compte de tout sauf de ce qui est écrit sur la page, comme si les mots se tenaient là pour exclure l'essentiel d'une vérité craintive; l'évocation d'Évangiles apocryphes signés Tubal, Sinistre et Philomèle (ou Imogène), l'annonce d'un nouveau Messie promettant d'apparaître chaque jeudi vers 16 heures, enfin une réinterprétation de la consubstantiation combinée tant bien que mal (plutôt mal) à un ensemble de règles pour la conservation du miel recopiées à la bibliothèque d'Alexandrie.

D'autres volent de succès en succès jusqu'au feu de joie final ; Cléobule trottine d'échec en échec en citant les Proverbes - le souvenir de saint Paul provoquant la mort d'un auditeur endormi par ses discours alors qu'il se tenait assis au bord de sa fenêtre, ce souvenir le console un petit peu, mais pas tous les soirs.

Il se prononce pendant des années sur des places de village, devant des foules toujours plus maigres, pressées de voir la fin: on dirait Molière au cours de ses premières tournées, sous la pluie, enfilant ses tragédies, roulant les $r$; il parle peut-être (Cléobule) de Dieu rétracté en lui-même pour faire de la place à l'univers créé, de Joseph jeté au fond d'une citerne, de "J'ai rendu mon visage semblable à un caillou" (Ésaïe, 50, 7), de "Tu faisais de ton dos comme une rue pour les passants" (Ésaïe, 51, 23), de "La trace du serpent sur la pierre" (Proverbes, 30, 19) - puis au moment de se lancer dans l'interprétation de Jérémie, "Ne suis-je pas aussi un Dieu de loin ?" $(23,23)$, il voit le dernier de ses auditeurs se lever pour aller traire une chèvre. Alors, devant un amphithéâtre de tabourets vides, il décide de simplifier ses discours, sa doctrine et sa foi par la même occasion, de quoi les rendre aussi évidentes, précisément, que ce lait de chèvre puisé en serrant le poing autour d'un pis - et le pis n'attendait que ça.

Être hérésiarque n'interdit pas d'être limpide, bien au contraire : il s'agit de soulever les foules, après tout, de frapper de stupeur ou d'ébranler immédiatement, convaincre à l'aide d'images limpides ou d'idées vraies, vraies d'une vérité véritable, sur le point d'atteindre la pureté des truismes. Cléobule s'en va partout, du sud au nord de l'Italie disant de Dieu qu'il est qui il est, selon le ego sum qui sum du Nouveau Testament ; puis il souligne l'équivalence du Dieu chrétien et de l'amour, pour mieux revendiquer Son amour de l'amour dans une formule ramassée (J'aime l'amour), dont saint Augustin 
s'inspirera des années plus tard, dans son livre le mieux vendu, le plus traduit, Les Confessions ("Amabam amare - J'aimais aimer"). Mais ça ne suffit pas: toujours les mêmes visages navrés, les tabourets vides, un public de sourds, d'assoupis, de siestes, ou de désœuvrés, et toujours une chèvre à traire en urgence - Cléobule se donne pour devoir d'avancer davantage vers l'épure, sinon l'épure de l'épure, pour obtenir une formule irréfutable sur le moment et à jamais, quelque chose comme Le bien est bien, d'où chacun déduirait, une fois rentré chez lui, la malignité du mal : Bonum, bonum est, plus tard encore réduit en un parfait Bonum, bonum, diamant irréductible qui était à deux doigts d'assurer son succès pour les siècles à venir. (Saint Augustin, immanquable plagiaire de génie, reprendra le Bonum, bonum de Cléobule dans ses Dissertations sur les Psaumes pour en faire une formule à peine améliorée : "Bonorum bonum, bonum est" perdant la concision au passage.)

Le bien du bien, il fallait y penser, c'était en vérité une idée de génie, comme il en advient rarement dans un siècle submergé par les caquetages : Bonum, bonum, en toute logique, et ceci dit du fond du cœur, aurait dû devenir aussitôt la devise du monde, des écoles jusqu'à la Sorbonne, traduite en langue peule et en espéranto, gravée sur des gaufrettes, reproduite en braille pour l'agrément des mal voyants, répétée sur un ton enthousiaste par des sauveurs de peuple, marchands de salut, militants pourfendeurs de toutes les afflictions, bonum, bonum, et repris par des foules faisant l'offrande, comme souvent, de leur conviction en remerciement de la vérité. Cléobule en avait même fait une chanson, il avait eu cette présence d'esprit, quelque chose comme :

Bonum

Bonum

Bonum, bonum, bonum

(etc.)

malheureusement, la mélodie n'a pas été conservée.

Il s'avance encore de l'actuelle Bari à l'actuelle Rimini, franchit les Alpes dans un sens puis dans l'autre, sans éléphant, sans attirer l'attention non plus; il tourne autour de l'actuelle Vérone en répétant bonum, bonum, accompagné au tambourin, mais l'Église ne semble toujours pas vouloir tenir compte de l'existence, quelque part, d'un Cléobule et de sa tendance à faire des schismes tous les dimanches.

Certaines mauvaises langues disent à son propos: il n'y croyait pas vraiment : la foi chrétienne modelée par Paul lui a toujours semblé être un vaste catalogue mal ordonné d'élucubrations, parfois splendides, parfois grotesques, parfois sublimes à force d'abstraction, et d'autres fois risibles pour les mêmes raisons, un bricolage accompli par des maîtres de la parole, laissant à leurs descendants (les suiveurs) le soin d'adapter leurs subtilités à un public avide de calme - cueilleur de simples. Pour Cléobule, malgré toute sa bonne volonté, les ulcères de Job, son énigmatique fidélité à Iaveh, Dieu à la fois omniprésent et disparu, l'amour divin traduit sous forme d'incendies, la grenade coupée en deux, le verbe fait chair, les clous de la croix et les écrits de Jésus du bout du doigt dans le sable, tout cela reste une arche de paroles démentes, comme si on avait rassemblé en urgence dans une même étable les représentants de six ou sept religions, certaines très folkloriques, dans l'espoir d'obtenir au bout du compte une sorte de syncrétisme (magiciens, géomètres, stratèges et somnambules).

L'idéal à ses yeux est une figure simple du panthéon païen, redorée chaque jour par des libations: la figure mineure de Danaé : pas beaucoup d'adorateurs pour elle, peu de lieux de culte, pas encore d'images, une discrétion agréable quand on veut lui adresser 
ses prières en toute tranquillité sans avoir à se frotter à mille autres clients venus réclamer, comme on en voit à la porte des oracles. Et puis, Danaé, c'est une mortelle visitée par Jupiter sous la forme d'une pluie d'or, le ruissellement d'une richesse pure venue d'en haut sans autre origine que le bon plaisir d'un seul dieu, sans autre nécessité sinon celle de pleuvoir et de faciliter ensuite des noces, toute sorte de réjouissances sur un lit de plume: au moment de célébrer son culte, Cléobule n'a aucune difficulté à se supposer (il s'agit là d'un exercice spirituel) à la fois dans la peau de Danaé et dans celle de Jupiter - Jupiter aurifère mais suffisamment anthropomorphe pour bien faire. Alors, le danaïsme, ce serait quoi? la pâmoison sans honte ni justification, sans le bruxisme de saint Paul; l'horizontalité d'un lit où les choses délicieuses adviennent, et demeurent mystérieuses.

Une journée fraîche de mars 137, Cléobule se prépare (et il murmure "enfin, enfin", à qui veut l'entendre) pour son bûcher d'hérésiarque : il y aura du monde et on viendra de loin, il y aura des chroniqueurs, des témoins de qualité, un nonce apostolique, des représentants et des messagers, des historiens de l'école Thucydide et une large portion du peuple rassemblée tout autour pour servir de public applaudissant - de la paille, des brindilles, des rondins et la poix, un prêtre sur la pointe des pieds pour demander au fautif de renoncer à ses erreurs (en vain, il le sait, mais à chacun ses répliques). Bien sûr, personne n'est au courant, personne ne viendra, les bûchers de l'Église romaine n'ont pas cours et Cléobule prévoit seulement de se brûler en effigie : voilà pourquoi depuis le matin, il s'applique à forger un mannequin à son image : il le forge, comment dire ? le cloue, le tricote, le tisse, le martèle, le modèle, le pétrit, le masse, tripotant son moi de terre glaise, et puis le chantourne, le maroufle, le tapisse, l'assemble, le poigne, le dorlote et le couvre de talc, tout cela à la fois, l'essentiel étant d'obtenir une ressemblance exacte, de quoi lui rendre justice à lui, le beau martyr : engendrer des fumées à son image. On a vu pendant des heures Cléobule dans l'attente de son apothéose manipuler le plâtre pour finir son autoportrait, puis vérifier dans un miroir l'avancée de son œuvre, de minute en minute, si soucieux du travail bien fait qu'en vérité, en vérité, il ne quittait jamais le miroir des yeux.

En 142 il prophétise une éclipse totale de Soleil advenue deux ans plus tôt, en comptant sur l'incertitude des calendriers, sur la confusion des esprits; en 145 une foule d'exaltés, du côté du Testaccio, s'empare de lui puis le jette au fleuve après avoir entrelacé au-dessus de son corps agité comme celui d'un chat maigre paroles de prières et paroles de conjuration. C'était un malentendu, une histoire de vin frelaté, mais Cléobule a cru ou a bien voulu croire mourir en sauveur d'humanité, adoré et conspué à la fois (de la même façon, en 44 avant Jésus-Christ, Cinna le poète est mort pour payer les crimes de Cinna le conjuré). 